\title{
ORIENTATIONAL TRANSITION IN THE CHOLESTERIC LAYER INDUCED BY ELECTRICALLY CONTROLLED IONIC MODIFICATION OF THE SURFACE ANCHORING
}

\author{
Vitaly S. Sutormin ${ }^{\mathrm{a} *}$, Ivan V. Timofeev ${ }^{\mathrm{a}, \mathrm{b}}$, Mikhail N. Krakhalev ${ }^{\mathrm{a}, \mathrm{b}}$, Oxana O. \\ Prishchepa ${ }^{\mathrm{a}, \mathrm{b}}$, and Victor Ya. Zyryanov ${ }^{\mathrm{a}}$ \\ ${ }^{a}$ Kirensky Institute of Physics, Krasnoyarsk Scientific Center, Siberian Branch of Russian Academy of \\ Sciences, Krasnoyarsk 660036, Russia; ${ }^{b}$ Siberian Federal University, Krasnoyarsk 660041, Russia
}

A reorientation of cholesteric liquid crystal with a large helix pitch induced by the electrically controlled ionic modification of the surface anchoring has been studied. In initial state, the cholesteric helix is untwisted completely owing to the normal surface anchoring specified by the cations adsorbed at the substrates. As a result, the homeotropic director configuration is observed within the cell. Under the action of dc electric field, one of the substrates becomes free from the layer of surface active cations, therefore, setting the planar surface anchoring. The latter, in turn, leads to the formation of the hybrid chiral structure. The threshold value and dynamic parameters have been estimated for this process as well as the range of control voltages, which do not allow the electrohydrodynamic instabilities. The twisted hybrid director configuration observed in the experiment has been analyzed by means of the simulation of polarization change of light propagating through the cholesteric layer with asymmetric (planar and homeotropic) surface anchoring on the cell substrates.

Keywords: cholesteric, ionic surfactant, anchoring transition, twisted hybrid structure, electrooptics

\section{Introduction}

Cholesteric liquid crystals (CLCs) are of great interest in various applications such as displays, light modulators, switchable diffraction gratings, liquid-crystal lasers and others [1-5]. This is determined by specific optical properties resulting from the helical ordering of director [6]. Vast majority of the devices are operated by the Frederiks effect inducing the director reorientation within the CLC bulk under the electric field. In these applications, the cholesteric is generally placed between two flat substrates specially treated to specify a certain surface anchoring of liquid crystal (LC) at the interface, which stays invariable during the process of director reorientation inside the cell bulk. 
Besides, the LC reorientation can also be caused by the anchoring transition under the influence of external factors such as temperature, UV-radiation or electric field [7-13]. Study of the director reorientation induced by the surface anchoring modification is useful for both the basic investigations and application, since the LC orientational transitions, which are impossible under the Frederiks effect, can be realized.

The method of electrically controlled modification of the surface anchoring by using the ionic surfactants has been developed for the nematic LC droplets [14-17], nematic layers [18-20] and cholesteric droplets [21]. For the nematic layers, the transitions from the homeotropic structure into the homeoplanar director configuration [18,19] and from the homeoplanar configuration into the twist structure [20] were found and studied.

The ionic-surfactant method is based on the change of surface active ions concentration on the substrate under the action of dc electric field. The nematic is doped with the surfactant. The latter dissociates into ions, among which at least one is the surface active ion specifying, for example, the homeotropic anchoring [18]. The surface active ions are adsorbed at the substrates. If their concentration is enough, they form the layers which can screen the planar orienting effect of the substrates and stimulate the LC alignment perpendicularly to the substrates. Thus, the homeotropic director configuration is realized in the LC cell. Under the action of dc electric field, the ions dissolved in the LC shift to the appropriate electrodes. Therefore, the surface active ion concentration decreases at the one of substrates and the planar anchoring, proper to the orienting coating, is restored here. As a result, the transition into the homeoplanar director configuration occurs within the LC cell.

It is obvious that the orientational transition results in the change of light transmission of the system consisting of the LC cell placed between the crossed polarizers. For example, in the initial state, the system transmission is close to zero because of the absence of birefringence in the LC layer with homeotropic orientational structure. However, when the director is reoriented into the homeoplanar configuration, the light transmission increases, in case the orientation of LC at the substrate with planar anchoring mismatches any polarizers.

This paper is devoted to the development of the ionic-surfactant method to control the director orientation of the cholesterics with a large helix pitch.

\section{Materials and experimental techniques}

The experiment was carried out with sandwich-like cells. These cells consisted of two glass substrates with transparent ITO electrodes coated with polymer films and the cholesteric layer between them. The polymer films based on the polyvinyl alcohol (PVA) doped with glycerin compound $(\mathrm{Gl})$ in the weight ratio PVA $: \mathrm{Gl}=1: 0.383$ were used as orienting coatings. The 
polymer films were deposited by spin coating and rubbed. The cell gap thickness $d$ was set using teflon spacers and measured by means of the interference method [22] with the spectrometer. Cholesteric mixtures were prepared using the nematic 4-pentyl-4'-cyanobiphenyl (5CB) with positive dielectric anisotropy $\Delta \varepsilon$ and the chiral additive cholesterylacetate $(\mathrm{ChA})$ in the weight ratios $5 \mathrm{CB}$ : ChA from $1: 0.0030$ to $1: 0.0155$, respectively. The nematic was preliminary doped with ionic surfactant cethyltrimethylammonium bromide (CTAB) in the weight ratio $5 \mathrm{CB}: \mathrm{CTAB}=1: 0.002$. $\mathrm{CTAB}$ dissolved in liquid crystal dissociates into the positively charged surface active ions $\mathrm{CTA}^{+}$and the negative ions $\mathrm{Br}^{-}$. The helical twisting power $H T P=6.5 \mu \mathrm{m}^{-1}$ of the additive $\mathrm{ChA}$ in the nematic $5 \mathrm{CB}$ was determined with the droplet method [23]. The used mixtures had pitch $10 \leq p \leq 51 \mu \mathrm{m}$ as calculated from $p=1 /\left(\operatorname{HTP} \times c_{w}\right)$, where $C_{w}$ is weight concentration of the chiral agent. The confinement ratio $\rho=d / p$ in LC cells was $0.16 \leq \rho \leq 0.85$.

The optical textures of the LC layer and their transformations under the electric field were analyzed in the crossed polarizers by means of the polarizing optical microscope (POM). Electro-optical response of the LC cell placed between crossed polarizers was studied with HeNe laser $(\lambda=632.8 \mathrm{~nm})$. The laser beam passed sequentially through the polarizer, the LC cell, the analyzer, and was detected by the photodiode. The LC cell was placed so that the angle between the substrates rubbing direction and the polarizer was $\alpha=45^{\circ}$ or $0^{\circ}$. The transmittance of the system was determined as $T=\left(I_{t} / I_{0}\right) \times 100 \%$, where $I_{0}$ is light intensity after the polarizer, $I_{t}$ is intensity after the analyzer.

\section{Results and discussion}

Figure 1 demonstrates the change of optical texture of the cholesteric layer doped with the ionic surfactant under the action of dc electric field. In the given LC cell, the confinement ratio $\rho$ is 0.4 . When the electric field is switched off, the optical texture of the LC layer in crossed polarizers is a uniform dark area (Figure 1a) independently of the sample rotation on the microscopic stage. Such an optical texture remains invariable until the $\mathrm{U}=2.3 \mathrm{~V}$. The latter was accompanied with the light transmission increase. In the range of control voltages $2.3 \mathrm{~V} \leq \mathrm{U} \leq 3.4 \mathrm{~V}$ the optical texture of the cell is a uniform bright area (Figure $1 \mathrm{~b}$ ). At that, the sample rotation relative to the crossed polarizers does not lead to the dark texture. The optical textures shown in Figures 1b, 1c prove this. Here the angle $\alpha$ between the rubbing direction and polarizer is $45^{\circ}$ and $0^{\circ}$, respectively. Besides, at $\alpha=0^{\circ}$ (Figure 1c) the dark texture is not obtained either under the analyzer rotation. At the control voltage $\mathrm{U}=3.5 \mathrm{~V}$ the domains began to form in the LC cell and they appear clearly at $\mathrm{U}=3.7 \mathrm{~V}$ (Figure 1d). 
The similar changes of optical texture were observed within the LC cells with $\rho=0.16$ and 0.85 approximately under the same control voltage. The only difference of the initial texture of cholesteric layer with $\rho=0.85$ is the presence of a small number of cholesteric bubbles $[24,25]$. However, after the application of ac voltage the cholesteric bubbles disappear and the optical texture of LC layer is the uniform dark area.

The optical texture of the LC layer in the absence of electric field (Figure 1a) and its invariability at the sample rotation relative to the crossed polarizers indicates a complete untwisting of the cholesteric helix and the formation of the homeotropic director orientation within the cell due to the ionic surfactant CTAB added (Figure 2a). The cholesteric helix untwisting occurs as an effect of the normal anchoring of LC molecules with the substrates [26].

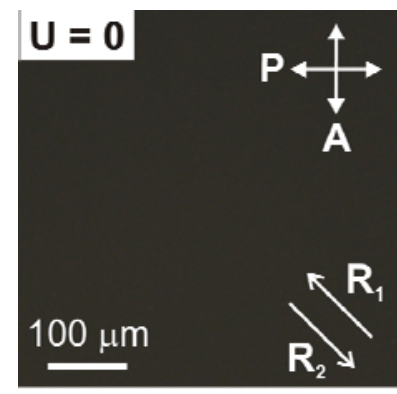

(a)

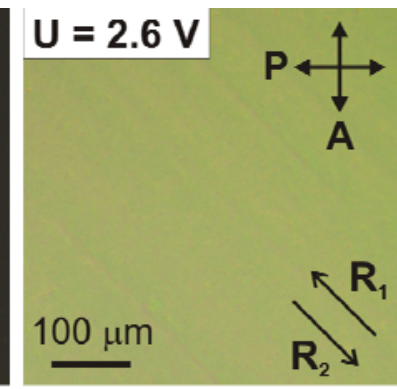

(b)

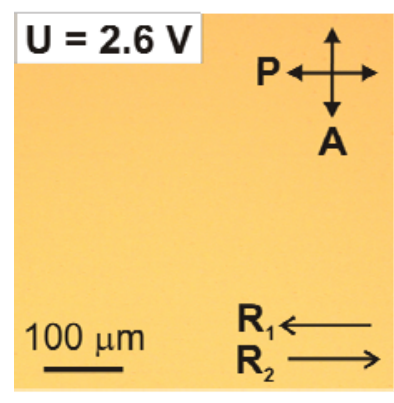

(c)

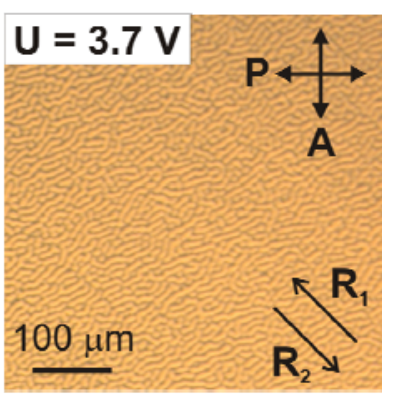

(d)

Figure 1. POM images of the optical textures of the cholesteric layer doped with the ionic surfactant made under different control voltages $U$ and $\alpha$ angles between the rubbing direction of the bottom substrate $\left(\mathbf{R}_{\mathbf{1}}\right)$ and the polarizer (P): (a) $\mathrm{U}=0 \mathrm{~V}, \alpha=45^{\circ}$; (b) $\mathrm{U}=2.6 \mathrm{~V}, \alpha=45^{\circ}$; (c) $\mathrm{U}=2.6 \mathrm{~V}, \alpha=0^{\circ}$; (d) $\mathrm{U}=3.7 \mathrm{~V}$, $\alpha=45^{\circ}$. The cell gap thickness is $d=8.1 \mu \mathrm{m}$. Confinement ratio is $\rho=0.4$. $\mathbf{A}$ is the analyzer direction. $\mathbf{R}_{2}$ is the rubbing direction of the top substrate.

The formation of homeotropic or twisted structures within the cholesteric layer with rigid normal anchoring depends on the confinement ratio $\rho$. The transition threshold $\rho$ th value is defined by the equation [27]:

$$
\rho_{\text {th }}=K_{33} /\left(2 K_{22}\right) \text {, }
$$


where $K_{33}$ and $K_{22}$ are elastic constants of the bend and twist deformations, respectively. When $\rho<\rho_{t h}$, the helix pitch is completely untwisted inside the LC cell with rigid normal anchoring. The twist structure is formed at $\rho>\rho_{\text {th }}$. The typical materials have the threshold value $\rho_{t h}$ about 1 [28]. The samples under study have $\rho<1$, which makes the helix pitch completely untwisted in the initial state.

When the dc electric field is applied, the surface anchoring on the electrode-anode substrate is modified, and the planar anchoring proper to the polymer orienting coating is restored at this substrate. The modification of the boundary conditions results in the reorientation of the LC structure and, consequently, in the change of the optical texture of the LC cell in the crossed polarizers (Figure 1b). Since the considered optical texture does not get dark completely during the sample rotation on the microscope stage relative to the crossed polarizers (Figure 1b, c), it means that the twisted orientation structure is formed within the LC layer. Thus, the optical textures of the LC layer presented in Figure 1b,c correspond to the hybrid aligned cholesteric [29-33]. Its orientational structure is shown in Figure 2b. It should be emphasized that the observable changes of optical texture cannot be induced by the Frederiks effect because the LC used in our experiment has positive dielectric anisotropy. In this case, the Frederiks effect could result only in the stabilization of the initial homeotropic director configuration.

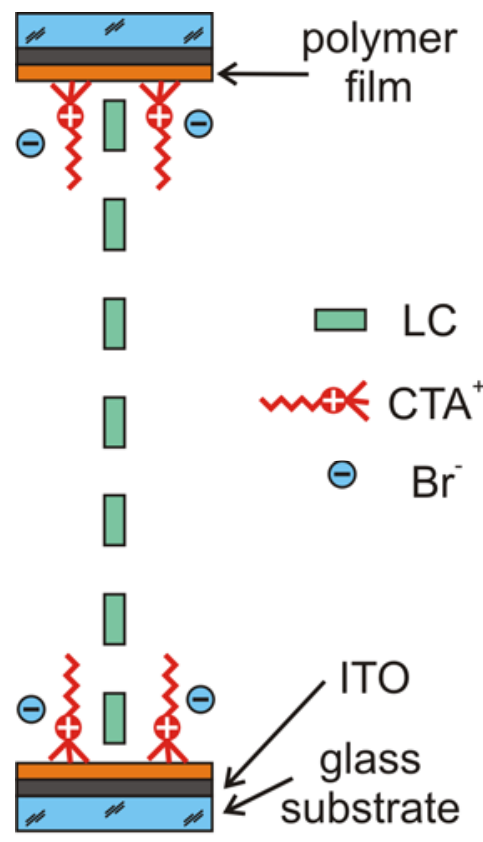

(a)

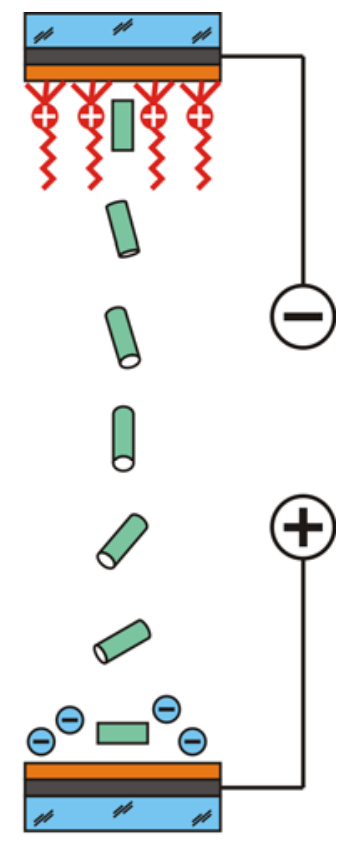

(b)

Figure 2. Scheme of the orientational transition induced by the dc electric field inside the LC cell filled with the CLC doped by the ionic surfactant. (a) the homeotropically aligned LC layer under the electric field off. (b) the cholesteric layer with a hybrid structure formed owing to the homeotropic-planar change of surface anchoring on the electrode-anode substrate. 
As mentioned above, the optical texture (Figure 1c) does not become dark completely under the analyzer rotation when the polarization direction of incident light coincides with the substrate rubbing direction. It indicates that the light after passing through the layer of hybrid aligned CLC is no longer linearly polarized. The light polarization change for such a system has been simulated. Firstly, the cholesteric orientational structure within the cell was calculated by means of the energy minimization of elastic deformations of the director field [34] under asymmetric boundary conditions. The LC surface anchoring on one of the substrates was planar and on the other one was homeotropic. Then, applying the Berreman $4 \times 4$-matrix method [35], the light polarization after the LC layer transmission was simulated. The following parameters of $5 \mathrm{CB}$ were used for the calculations: the elastic constants of the splay deformation $K_{11}=5.7 \mathrm{pN}$, the twist deformation $K_{22}=3.5 \mathrm{pN}$ and the bend deformation $K_{33}=7.7 \mathrm{pN}$ [36]; the extraordinary and ordinary refractive indices $n_{\mathrm{e}}=1.7002, n_{\mathrm{o}}=1.5294(\lambda=632.8 \mathrm{~nm})$, respectively [37]; the thickness of LC layer $d=8.1 \mu \mathrm{m}$, the helix pitch $p=21 \mu \mathrm{m}$, the confinement ratio $\rho=0.4$.

Figure 3 shows the calculation data for the hybrid aligned cholesteric structure. The simultaneous tilt and twist of the director are seen to occur inside the LC layer. The total twist angle of director is $98^{\circ}$. The Berreman method simulation has revealed that the linearly polarized light beam $(\lambda=632.8 \mathrm{~nm})$ passing through such a LC orientational structure gets elliptically polarized. The ratio of semi-major axis to semi-minor axis of the polarization ellipse is 3.8. At that, the $\beta$ angle between the polarization direction of incident light and the semi-major axis of elliptically polarized light is $68^{\circ}$ when leaving the LC layer.

We measured $\beta$ angle depending on the control voltage value. The cell was placed between the crossed polarizers so that the rubbing direction of input substrate coincided with the polarization direction of incident He-Ne laser beam. After that, the dc voltage was applied to the LC cell. When the light transmission reached the saturation, which corresponds to the formation of the hybrid aligned CLC, the minimal light transmission of the system was found by means of the analyzer rotation. In this case, the direction of semi-major axis of the elliptically-polarized light was perpendicular to the analyzer direction. In the range $2.6-3.2 \mathrm{~V}$ of control voltages $\beta$ angle changed from $62^{\circ}$ to $64^{\circ}$, respectively. These data are in a good accordance with the calculated results. 


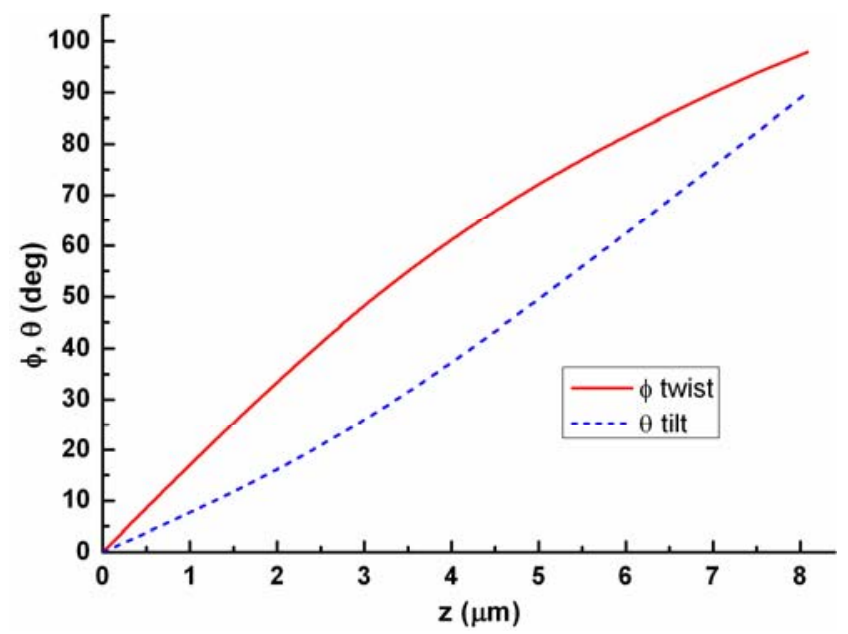

Figure 3. Calculation data of tilt angle $\theta$ and twist angle $\varphi$ of the director versus the $z$-coordinate perpendicular to the cell substrates for the structure of hybrid aligned cholesteric. $z=0$ and $z=8.1 \mu \mathrm{m}$ correspond to the substrates with planar and homeotropic surface anchoring, respectively. Thickness of the LC layer is $d=8.1 \mu \mathrm{m}$. The helix pitch is $p=21 \mu \mathrm{m}$.

Figure 4 shows the oscillograms of the square-wave response of the LC cell placed between crossed polarizers. The pulse amplitude is $2.6 \mathrm{~V}$ and its duration is $10 \mathrm{~s}$. The oscillogram in Figure $4 \mathrm{a}$ corresponds to the situation, when the polarization of incident light coincides with the rubbing direction $\left(\alpha=0^{\circ}\right.$ ), and $\alpha=45^{\circ}$ for Figure $4 \mathrm{~b}$. In the initial state, the light transmission of the system is close to zero because of the homeotropic director orientation. The applied electric pulse increases the light transmission caused by the formation of the hybrid aligned cholesteric. If the polarization of incident light coincides with the rubbing direction (Figure 4a), the light transmission saturation reaches $58 \%$. The on-time $\tau_{\text {on }}$ defined as the interval between the rising edge of electric pulse and the increase of the transmittance up to $90 \%$ from the saturation value was $0.31 \mathrm{~s}$. The off-time $\tau_{\text {off }}$ defined as the interval between the falling edge of electric pulse and the decrease of the transmittance down to $10 \%$ of the saturation value was $0.51 \mathrm{~s}$. For $\alpha=45^{\circ}$ the transmittance saturation reached $51 \%$ (Figure $4 \mathrm{~b}$ ), $\tau_{\mathrm{on}}=0.13 \mathrm{~s}, \tau_{\mathrm{off}}=0.59 \mathrm{~s}$. Under the control voltages $2.6 \leq \mathrm{U} \leq 3.2 \mathrm{~V}$, the on-time $\tau_{\text {on }}$ did not practically change. The off-time $\tau_{\text {off }}$ in the same range of voltages increased up to $1.75 \mathrm{~s}$. It should be noted that the change of the control pulse form is able to lead to a significant improvement of the dynamic characteristics of LC cell with electrically controlled ionic modification of the surface anchoring [20]. 

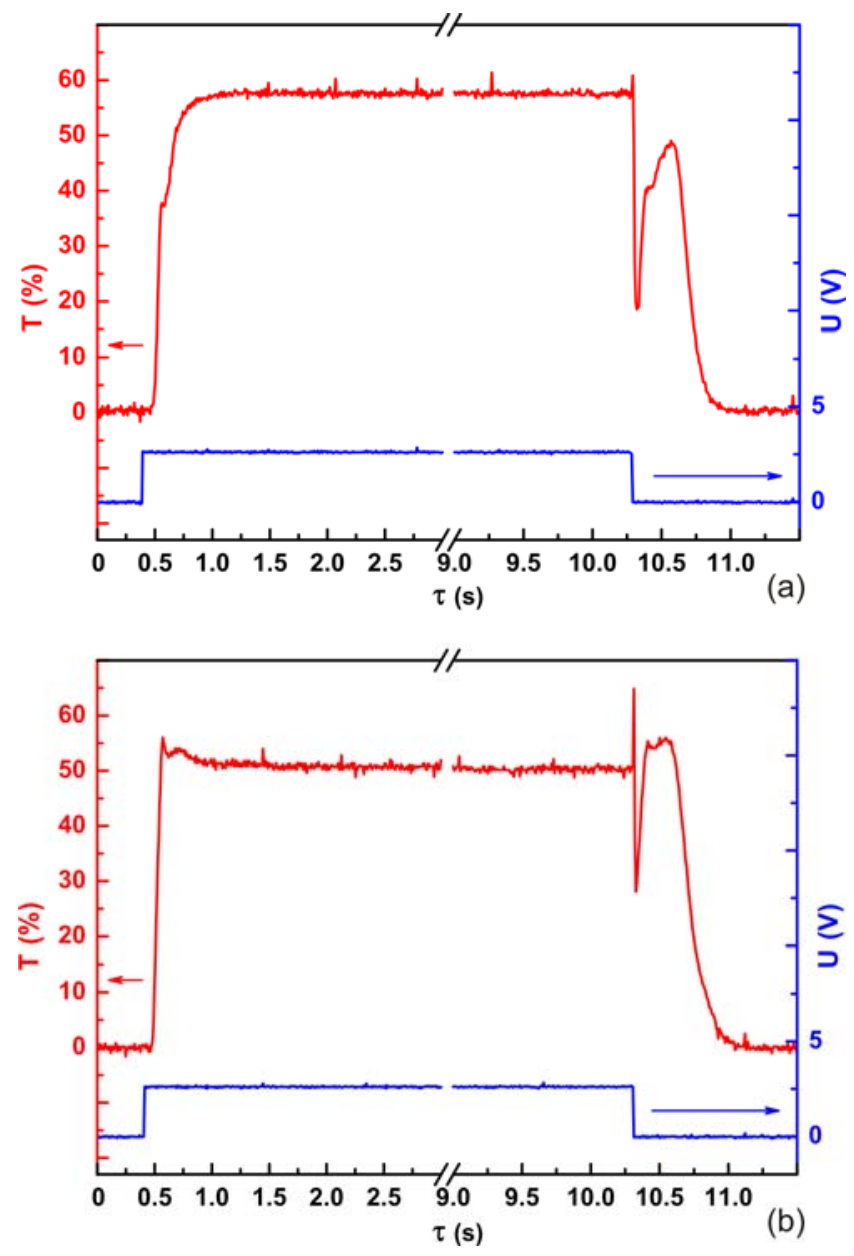

Figure 4. Optical response of the LC cell filled with the ionic surfactant-doped cholesteric in crossed polarizers at different $\alpha$ angles between the polarizer and the rubbing direction: (a) $\alpha=0^{\circ}$; (b) $\alpha=45^{\circ}$. Applied voltage amplitude is $2.6 \mathrm{~V}$. Confinement ratio is $\rho=0.4$.

\section{Conclusions}

The reorientation of cholesteric LC caused by electrically controlled ionic modification of the surface anchoring has been studied in the present paper. The reorientation has been examined for the cholesteric with the large helix pitch $(10 \mu \mathrm{m}-51 \mu \mathrm{m})$, and the ratio of the LC layer thickness to the pitch changed in the range $0.16-0.85$. It was shown that in the LC cell the dc electric field induces the transition from the homeotropic director configuration into the twisted hybrid one. The director reorientation is found to have the threshold at $2.3 \mathrm{~V}$. It proceeds without electrohydrodynamical instabilities under the control voltages $2.3 \mathrm{~V} \leq \mathrm{U} \leq 3.4 \mathrm{~V}$. On-time for the LC cells was tenth parts of a second and off-time was about a second.

To verify the experimental results we simulated the orientational structure of hybrid aligned cholesteric and the change of linearly-polarized light after passing the LC layer. A situation when the polarization of incident light coincides with the director at the planar anchoring substrate was considered. The calculations show that the light passing through the hybrid aligned cholesteric layer becomes elliptically polarized, and the semi-major axis of ellipse turns relative 
to the initial polarization. The simulated rotation angle is in a good agreement with the experimental value. This confirms the formation of the twisted hybrid director configuration under the dc electric field applied to the LC cell.

Thus, the ionic modification of surface anchoring initiates the nematic-cholesteric transition similar to the transition induced by the Frederiks effect inside the LC cell filled with a cholesteric with negative dielectric anisotropy [38].

\section{Disclosure statement}

No potential conflict of interest was reported by the authors.

\section{Funding}

This work was supported by Russian Foundation for Basic Research (RFBR) under Grants [15-02-06924 and 16-53-00073] and by the Siberian Branch of the Russian Academy of Sciences under Complex Program [II.2P 0358-2015-0010]. V.S.S. acknowledges financial support from RFBR under Grants [16-32-60036 and 16-32-00164]. 


\section{References}

[1] Yang D-K, Huang X-Y, Zhu Y-M. Bistable cholesteric reflective displays: materials and drive scheme. Annu Rev Mater Sci. 1997;27:117-146. doi:10.1146/annurev.matsci.27.1.117.

[2] $\mathrm{Xu} \mathrm{M}$, Yang D-K Dual frequency cholesteric light shutters: Appl Phys Lett. 1997;70:720-722. doi:10.1063/1.118261.

[3] Subacius D, Bos PJ, Lavrentovich OD. Switchable diffractive cholesteric gratings. Appl Phys Lett. 1997;71:1350-1352. doi:10.1063/1.119890.

[4] Kopp VI, Fan B, Vithana KM, et al. Low-threshold lasing at the edge of a photonic stop band in cholesteric liquid crystals. Opt Lett. 1998;23:1707-1709. doi:10.1364/OL.23.001707.

[5] Moreira MF., Carvalho ICS, Cao W. Cholesteric liquid-crystal laser as an optic fiberbased temperature sensor. Appl Phys Lett. 2004;85:2691-2693. doi:10.1063/1.1781363.

[6] Blinov LM, Chigrinov VG. Electrooptics Effects in Liquid Crystal Materials. New York: Springer; 1994.

[7] Ryschenkow G, Kleman M. Surface defects and structural transitions in very low anchoring energy nematic thin film. J Chem Phys. 1976;64:404-412. doi:10.1063/1.431934.

[8] Ichimura K, Suzuki Y, Seki T et al. Reversible change in alignment mode of nematic liquid crystals regulated photochemically by "command surfaces" modified with an azobenzene monolayer. Langmuir. 1988;4:1214-1216. doi:10.1021/la00083a030.

[9] Petrov AG, Durand G. Electric field transport of biphilic ions and anchoring transitions in nematic liquid crystals. Liq Cryst. 1994;17:543-554. doi:10.1080/02678299408036739.

[10] Komitov L, Helgee B, Felix J, et al. Electrically commanded surfaces for nematic liquid crystal displays. Appl Phys Lett. 2005;86:023502. doi:10.1063/1.1849844.

[11] Kim KJ, Araoka F, Jeong SM, et al. Bistable device using anchoring transition of nematic liquid crystals. Appl Phys Lett. 2009;95:063505. doi:10.1063/1.3202781.

[12] Kumar TA, Le KV, Aya S, et al. Anchoring transition in a nematic liquid crystal doped with chiral agents. Phase Trans. 2012;85:888-899. doi:10.1080/01411594.2012.692092.

[13] Kundu S, Kang S-W. Photo-stimulated phase and anchoring transitions of chiral azo-dye doped nematic liquid crystals. Opt Express. 2013;21:31324-31329. doi:10.1364/OE.21.031324.

[14] Zyryanov VY, Krakhalev MN, Prishchepa OO et al. Orientational structure transformations caused by the electric-field-induced ionic modification of the interface in 
nematic droplets. J Exp Theor Phys Lett. 2007;86:383-388. doi:10.1134/S0021364007180087.

[15] Zyryanov VY, Krakhalev MN, Prishchepa OO et al. Inverse regime of ionic modification of surface anchoring in nematic droplets. J Exp Theor Phys Lett. 2008;88:597-601. doi:10.1134/S002136400821011X.

[16] Zyryanov VY, Krakhalev MN, Prishchepa OO. Texture transformation in nematic droplets caused by ionic modification of boundary conditions. Mol Cryst Liq Cryst. 2008;489:273/[599]-279/[605]. doi:10.1080/15421400802219189.

[17] Krakhalev MN, Prishchepa OO, Zyryanov VY. Inverse mode of ion-surfactant method of director reorientation inside nematic droplets. Mol Cryst Liq Cryst. 2009;512:152/[1998]-157/[2003]. doi:10.1080/15421400903050814.

[18] Sutormin VS, Krakhalev MN, Prishchepa OO et al. Electrically controlled local Frédericksz transition in a layer of a nematic liquid crystal. J Exp Theor Phys Lett. 2012;96:511-516. doi:10.1134/S0021364012200131.

[19] Sutormin VS, Krakhalev MN, Zyryanov VY. The dynamics of the response of an electrooptic cell based on a nematic layer with controlled surface anchoring. Tech Phys Lett. 2013;39:583-586. doi:10.1134/S1063785013070134.

[20] Sutormin VS, Krakhalev MN, Prishchepa OO et al. Electro-optical response of an ionicsurfactant-doped nematic cell with homeoplanar-twisted configuration transition. Opt Mater Express. 2014;4:810-815. doi:10.1364/OME.4.000810.

[21] Gardymova AP, Zyryanov VY, Loiko VA. Multistability in polymer-dispersed cholesteric liquid crystal film doped with ionic surfactant. Tech Phys Lett. 2011;37:805808. doi: 10.1134/S1063785011090094.

[22] Huibers PDT, Shah DO. Multispectral determination of soap film thickness. Langmuir. 1997;13:5995-5998. doi:10.1021/1a960738n.

[23] Candau S, Le Roy P, Debeauvais F. Magnetic field effects in nematic and cholesteric droplets suspended in a isotropic liquid. Mol Cryst Liq Cryst. 1973;23:283-297. doi:10.1080/15421407308083378.

[24] Kawachi M, Kogure O, Kato Y. Bubble domain texture of a liquid crystal. Jpn J Appl Phys. 1974;13:1457-1458. doi:10.1143/JJAP.13.1457.

[25] Pirkl S, Ribiere P, Oswald P. Forming process and stability of bubble domains in dielectrically positive cholesteric liquid crystals. Liq Cryst. 1993;13:413-425. doi:10.1080/02678299308026314.

[26] Harvey TB. A boundary induced cholesteric-nematic phase transition. Mol Cryst Liq Cryst. 1977;34:225-229. doi:10.1080/15421407708083711. 
[27] Zel'dovich BY, Tabiryan NV. Freedericksz transition in cholesteric liquid crystals without external fields. Sov Phys J Exp Theor Phys Lett. 1981;34:406-408.

[28] Oswald P, Baudry J, Pirkl S. Static and dynamic properties of cholesteric fingers in electric field. Phys Rep. 2000;337:67-96. doi:10.1016/S0370-1573(00)00056-9.

[29] Dozov I, Penchev I. Structure of a hybrid aligned cholesteric liquid crystal cell. J Phys France. 1986;47:373-377. doi:10.1051/jphys:01986004703037300.

[30] Lewis MR, Wiltshire CK. Hybrid aligned cholesteric: a novel liquid-crystal alignment. Appl Phys Lett. 1987;51:1197-1199. doi:10.1063/1.98731.

[31] Belyaev SV, Barnik MI, Beresnev GA, et al. Optical and electrooptical properties of homeoplanar layers of cholesteric liquid crystals. Liq Cryst. 1988;3:1279-1282. doi:10.1080/02678298808086585.

[32] Hsiao Y-C, Timofeev IV, Zyryanov VY, et al. Hybrid anchoring for a color-reflective dual-frequency cholesteric liquid crystal device switched by low voltages. Opt Mater Express. 2015;5:2715-2720. doi:10.1364/OME.5.002715.

[33] Ryabchun A, Bobrovsky A, Stumpe J, et al. Rotatable diffraction gratings based on cholesteric liquid crystals with phototunable helix pitch. Adv Opt Mater. 2015;3:12731279. doi:10.1002/adom.201500159.

[34] Timofeev IV, Lin Y-T, Gunyakov VA, et al. Voltage-induced defect mode coupling in a one-dimensional photonic crystal with a twisted-nematic defect layer. Phys Rev E. 2012;85:011705. doi:10.1103/PhysRevE.85.011705.

[35] Berreman DW. Optics in stratified and anisotropic media: 4×4-matrix formulation. J Opt Soc Am. 1972;62:502-510. doi:10.1364/JOSA.62.000502.

[36] Cui M, Kelly JR. Temperature dependence of visco-elastic properties of 5CB. Mol Cryst Liq Cryst. 1999;331:49-57. doi:10.1080/10587259908047499.

[37] Bunning JD, Crellin DA, Faber TE. The effect of molecular biaxiality on the bulk properties of some nematic liquid crystals. Liq Cryst. 1986;1:37-51. doi:10.1080/02678298608086488.

[38] Smalyukh II, Senyuk BI, Palffy-Muhoray P, et al. Electric-field-induced nematiccholesteric transition and three-dimensional director structures in homeotropic cells. Phys Rev E. 2005;72:061707. doi:10.1103/PhysRevE.72.061707. 